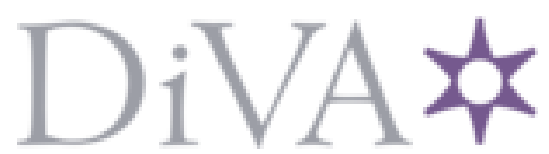

http://www.diva-portal.org

This is the published version of a paper presented at IEEE Intelligent Vehicles Symposium (IV).

Citation for the original published paper:

Lima, P F., Nilsson, M., Trincavelli, M., Mårtensson, J., Wahlberg, B. (2016)

Experimental evaluation of economic model predictive control for an autonomous truck.

In:

N.B. When citing this work, cite the original published paper.

Permanent link to this version:

http://urn.kb.se/resolve?urn=urn:nbn:se:kth:diva-200340 


\title{
Experimental Evaluation of Economic Model Predictive Control for an Autonomous Truck
}

\author{
Pedro F. Lima ${ }^{1}$, Marco Trincavelli ${ }^{2}$, Mattias Nilsson ${ }^{2}$, \\ Jonas Mårtensson ${ }^{1}$, and Bo Wahlberg ${ }^{1}$
}

\begin{abstract}
In this paper, we propose a controller for smooth autonomous path following. The controller is formulated as an economic model predictive controller. The economic cost introduced in the objective function leads to a smooth driving, since we minimize the first and second derivatives of the curvature function (i.e., we encourage linear curvature profiles). Since the curvature in clothoids varies linearly with the path arc-length, we use the smoothness and comfort characteristics of clothoid-driving to obtain a compact and intuitive controller formulation. We enforce convergence of the controller to the reference path with soft constraints that avoid deviations from the reference path. Finally, we present real life experiments where the controller is deployed on a Scania construction truck that show that the proposed controller outperforms a purepursuit controller. Moreover, we detail how the few tuning parameters can affect the obtained solution in practice.
\end{abstract}

\section{INTRODUCTION}

The enormous demand for more efficient transportation systems and increased need for goods creates congestions, as well as safety and environmental concerns. Autonomous vehicles have emerged to fulfill the need of more efficient and safer systems. In the past five years, many major vehicle manufacturers, suppliers and technology companies have started projects and collaborations around the autonomous vehicles theme. The automotive and technological industry are far closer than expected to produce a commercial autonomous vehicle [1]-[4]. The idea of developing such systems is not new and there have been different competitions and challenges since the '90s to demonstrate and compare autonomous vehicles capabilities. It has been over 10 years since the first DARPA Grand Challenges in 2004 and 2005 demonstrated the feasibility of autonomous driving [5].

One of the main challenges of autonomous vehicles is the lateral control of the vehicle. While most research and industry development is targeting urban related autonomous driving (e.g., [6], [7]), we research control methods for high-precision off-road path following. The idea is that the development of autonomous vehicles with specialized applications (e.g., mining sites) is facilitated by the lower requirements in terms of investment, infrastructure expansion and law adaptation, especially when compared to urban driving. Furthermore, eliminating the human-in-the-loop factor we expect to eliminate the number of human fatalities and increase productivity and efficiency in these working sites. Autonomous trucks is a significant research area, since mining and construction is a big market segment, specific for trucks rather than for personal cars.

\footnotetext{
${ }^{1}$ Integrated Transport Research Lab and ACCESS Linnaeus Centre, Department of Automatic Control, KTH Royal Institute of Technology, SE-100 44 Stockholm, Sweden. pfrdal@kth.se, jonaslakth.se, boakth.se

${ }^{2}$ Research and Development, Scania CV AB, 15187 Södertälje, Sweden, firstname. secondnamedscania.com

This work has been funded by the Swedish government and automotive industry within the FFI program - Strategic Vehicle Research and Innovation under the project iQMatic 2012-04626.
}

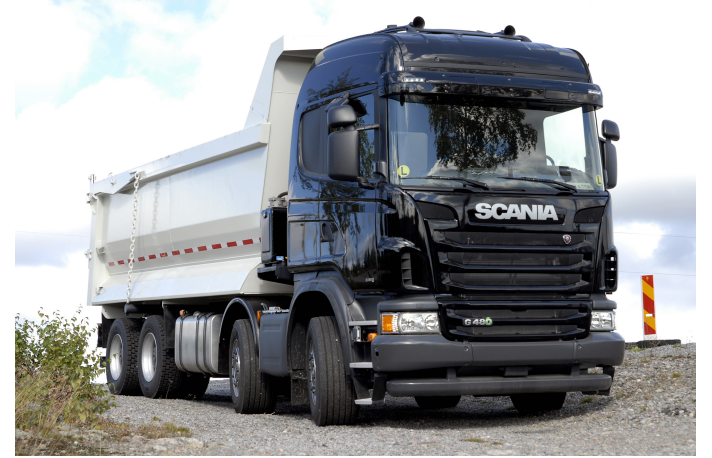

Fig. 1. Modified Scania G480 construction truck used as experimental and research platform.

MPC has been widely used to address the autonomous vehicle guidance problem [8]-[11]. In [8], an NMPC controller is presented in order to stabilize a vehicle along a desired path, while fulfilling its physical constraints. Since a nonlinear vehicle model is considered, the resulting MPC controller has a considerable computational burden, which makes it difficult to be implemented in real-time. MPC controllers limit experimental validation due to the use of complex models for predicting the vehicle behavior, which makes the optimization complex and slow. Also, adding constraints, such as imposing certain comfort measures, increases the problem complexity and the computational burden. To overcome these problems, a linear time-varying (LTV) MPC for active front steering (AFS) is proposed in [9]. There, the discrete time linear system is based on successive online linearizations of the nonlinear vehicle model, and the stability conditions for LTV-MPC controllers are also presented. A study about the use of kinematic and dynamic vehicle models for MPC control design is detailed in [10]. The authors show that the kinematic model has better forecast errors when discretized at $200 \mathrm{~ms}$, compared to $100 \mathrm{~ms}$. Therefore, the authors motivate the MPC design based on a kinematic vehicle model since it is simpler than the dynamical model and still considerably accurate. Economic MPC (EMPC) [12] is a recent variant of MPC, which was born to efficiently combine the hierarchical separation between economic and dynamic performance. Typically, an upper layer provides the optimal set-point (from the economic point of view) to the MPC, which is responsible for stabilizing the system and reject any disturbances (from the dynamical point of view). Although recent, it has been applied, for example, to building temperature and smart grid management [13], [14]. To our best knowledge, the EMPC framework has never been applied to autonomous driving.

The work presented here comes in line with our previous research. The concept of clothoids was used in the design of a linearly-time varying (LTV) clothoid-based model predictive 
controller (MPCC) [15]. We present an EMPC for lateral control of an autonomous vehicle. In contrast with our previous work, we do not need to compute a clothoidbased sparsified version of the reference path as in [15]. Also, while a clothoid model is used to predict the vehicle motion in the MPCC, we now use a kinematic vehicle model. In standard tracking MPC approaches, the cost function of the optimization problem is zero at the optimal reference trajectory. However, that is not the case in the EMPC due to the inclusion of an economic cost associated with the system operation [12]. In our case, the economic cost imposes smooth and comfortable driving. We explicitly include the vehicle curvature in the cost function to influence its shape and characteristics. To enforce convergence to the reference path, we also include the vehicle distance from the path. Currently, clothoids are the standard transition curve in road design [16]. In the case of ground vehicles, clothoidal road design provides a linear change in the vehicle steering angle when performing a turn, preventing sudden changes in lateral acceleration and therefore yielding low values of lateral jerk [17].

We demonstrate the EMPC performance in a real vehicle by deploying it on a Scania construction truck. We compare the performance of the EMPC with a pure-pursuit controller (PPC) that was previously implemented on the truck. We analyze the performance of the controllers in terms of path tracking accuracy.

The rest of this paper is organized as follows: in Section II, we address the problem of clothoid-based path following using an receding-horizon framework by developing an optimal lateral controller for a truck; in Section III, we benchmark the performance of the proposed controller against a pure-pursuit controller in real experiments using a Scania construction truck; finally, in Section IV, we provide some concluding remarks and outline future work.

\section{ECONOMIC MODEL PREDICTIVE CONTROLLER}

In this section, we address the problem of lateral control of an autonomous vehicle. We formulate an EMPC in order to combine path tracking accuracy and driving smoothness.

\section{A. Space-based kinematic vehicle model}

The movement of a car-like nonholonomic vehicle, such as a truck, a car or a bus, at low speeds (i.e., when the lateral dynamics have little influence) can approximately be described by its kinematics without significant loss of prediction accuracy [10]. Therefore, the mathematical equations describing the vehicle's position $(x, y)$, orientation $\theta$ and curvature $\kappa$ over the traveled distance $s$ are

$$
\begin{aligned}
& \frac{d x(s)}{d s}=\cos (\theta(s)) \\
& \frac{d y(s)}{d s}=\sin (\theta(s)) \\
& \frac{d \theta(s)}{d s}=\kappa(s) .
\end{aligned}
$$

Furthermore, the relation between the desired curvature and the desired vehicle steering angle can be described with a non-linear mapping.

We discretize the vehicle model (2) by letting the vehicle state at each sampling point be defined as $\mathbf{z}\left(s_{i}\right)=\mathbf{z}_{i}=\left[x_{i}, y_{i}, \theta_{i}\right]^{\top}$, and the vehicle input be $\kappa\left(s_{i}\right)=$ $\kappa_{i}$. The vehicle state is sampled every $\Delta s_{i}=s_{i+1}-s_{i}$, which is the traveled distance between two consecutive points.
The curvature is assumed to be constant between $\kappa\left(s_{i}\right)$ and $\kappa\left(s_{i+1}\right)$. The integral discretization of (2a) and (2b) is based on the fact that integrals can be approximated by Riemann sums [18]. Thus, the position $\left(x\left(\boldsymbol{\kappa}_{n}\right), y\left(\boldsymbol{\kappa}_{n}\right)\right)$, with $n<H$, where $H \in \mathbb{Z}^{+}$is the prediction horizon of the MPC, after applying $\boldsymbol{\kappa}_{n}=\left[\kappa_{0}, \ldots, \kappa_{n}\right]$ is described as

$$
\begin{aligned}
& x\left(\boldsymbol{\kappa}_{n}\right)=\sum_{i=0}^{n} \cos \left(\sum_{j=0}^{i} \kappa\left(s_{j}\right) \Delta s_{j}\right) \Delta s_{i} \\
& y\left(\boldsymbol{\kappa}_{n}\right)=\sum_{i=0}^{n} \sin \left(\sum_{j=0}^{i} \kappa\left(s_{j}\right) \Delta s_{j}\right) \Delta s_{i} .
\end{aligned}
$$

The equivalent vectorial version for $\kappa=\left[\kappa_{0}, \ldots, \kappa_{H}\right]$ is

$$
\begin{aligned}
& \mathbf{x}(\boldsymbol{\kappa})=\mathbf{S} \cos (\mathbf{S} \boldsymbol{\kappa}) \\
& \mathbf{y}(\boldsymbol{\kappa})=\mathbf{S} \sin (\mathbf{S} \boldsymbol{\kappa})
\end{aligned}
$$

where $\mathbf{S} \in \mathbb{R}^{(H+1) \times(H+1)}$ is a lower triangular matrix that performs the cumulative sum operation along a vector

$$
\mathbf{S}=\left[\begin{array}{ccccc}
\Delta s_{0} & & & & \\
\Delta s_{0} & \Delta s_{1} & & & \\
\Delta s_{0} & \Delta s_{1} & \Delta s_{2} & & \\
\vdots & \vdots & \vdots & \ddots & \\
\Delta s_{0} & \Delta s_{1} & \Delta s_{2} & \ldots & \Delta s_{l-1}
\end{array}\right]
$$

\section{B. Problem formulation}

Let a reference path be constituted by $N$ waypoints of the form $\mathbf{z}_{i}^{\text {ref }}=\left[x_{i}^{\text {ref }}, y_{i}^{\text {ref }}, \theta_{i}^{\text {ref }}, s_{i}\right]^{\top}, i=1, \ldots, N$ representing the path Cartesian coordinates, the orientation, and the distance traveled along the path since the first point, respectively.

Let the economic cost of the EMPC be interpreted as the smoothness (or the comfort) of the vehicle motion and its predictions. To achieve that, the economic cost is designed in order to penalize the first and the second-order derivative of the curvature function. The first-order derivative is related to the vehicle lateral jerk, for which high values are perceived by a human as uncomfortable. On the other hand, the second derivative of the curvature is related to the angular acceleration of the steering wheel. Therefore, it is desirable to maintain both the first and second derivatives as low as possible, while tracking the reference path in order to achieve a smooth driving. For instance, a truck designed for mining applications weights around 100 tons when loaded. As a consequence, a high curvature request change rate can seriously damage the tires and the vehicle's steering servo. The other goal of the EMPC is to accurately follow a given reference path. Therefore, soft constraints are added to the problem in order to avoid large deviations from the path. Moreover, the vehicle maximum curvature and change rate are also limited.

The EMPC is formulated as the following optimization problem: 


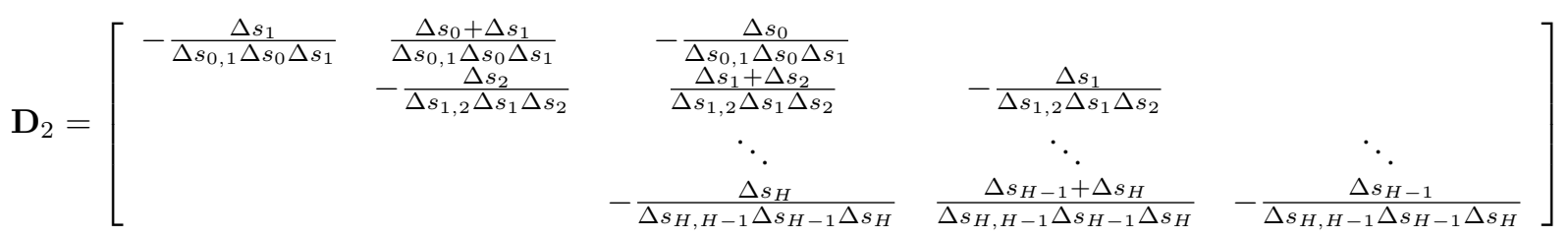

$$
\begin{array}{cl}
\min _{\kappa} & || \mathbf{D}_{2} \boldsymbol{\kappa}\left\|\left.\right|_{2} ^{2}+\alpha\right\| \mathbf{D}_{1} \boldsymbol{\kappa} \|_{2}^{2} \\
\text { s. t. } & \left|\mathbf{x}(\kappa)-\mathbf{x}_{\text {ref }}\right| \leq \boldsymbol{\varepsilon}_{x} \\
& \left|\mathbf{y}(\kappa)-\mathbf{y}_{\text {ref }}\right| \leq \varepsilon_{y} \\
& \left|\mathbf{D}_{1} \boldsymbol{\kappa}\right| \leq \mathbf{1} c_{\max } \\
& |\boldsymbol{\kappa}| \leq \mathbf{1} \kappa_{\max } \\
& \kappa_{0}=\kappa_{\text {vehicle }}
\end{array}
$$

where $\kappa \in \mathbb{R}^{H+1}$ is the curvature vector to be optimized, $\varepsilon_{x} \in \mathbb{R}^{H}$ and $\boldsymbol{\epsilon}_{y} \in \mathbb{R}^{H}$ are the maximum allowed deviations from the path waypoints in $x$ and $y$ directions, $\mathbf{D}_{2} \in \mathbb{Z}^{(H-1) \times(H+1)}$ and $\mathbf{D}_{1} \in \mathbb{Z}^{H \times(H+1)}$ are the matrix operators that calculate the second- and first-order differences of a vector, respectively, and $\alpha \in \mathbb{R}$ penalizes the curvature change rate magnitude. The constants $\kappa_{\max }$ and $c_{\max }$ denote the maximum curvature and change rate that the vehicle can perform, respectively, and $\mathbf{1}$ is a column vector filled with ones. Note that the coordinates of the clothoid-based path $(\mathbf{x}, \mathbf{y})$ are only dependent on the clothoid curvature $\kappa$, since we can accurately approximate the arclengths between points to be equal to the arc-lengths between the reference path waypoints. The inequalities (5b) to $(5 \mathrm{e})$ are performed element-wise and the equality constraint sets the initial curvature value of the optimization problem.

To calculate the second derivative of the curvature, we use a second-order difference operator, $\mathbf{D}_{2}$, weighted with the distance between the waypoints. The operator $\mathbf{D}_{2}$ used to approximate the second-order derivatives of the curvature is based on finite differences. The first derivative of the curvature function with respect to traveled distance is approximated as

$$
\left.\frac{\mathrm{d} \kappa(s)}{\mathrm{d} s}\right|_{s_{i}} \approx \frac{\kappa\left(s_{i+1}\right)-\kappa\left(s_{i}\right)}{\left|s_{i+1}-s_{i}\right|}=\frac{\kappa\left(s_{i+1}\right)-\kappa\left(s_{i}\right)}{\left|\Delta s_{i}\right|}
$$

and consequently the second discrete derivative is

$$
\begin{gathered}
\left.\frac{\mathrm{d}^{2} \kappa(s)}{\mathrm{d} s^{2}}\right|_{s_{i}} \approx \frac{\frac{\kappa\left(s_{i+1}\right)-\kappa\left(s_{i}\right)}{\Delta s_{i}}-\frac{\kappa\left(s_{i}\right)-\kappa\left(s_{i-1}\right)}{\Delta s_{i-1}}}{\Delta s_{i-1, i}} \\
=\frac{\left(\kappa\left(s_{i+1}\right)-\kappa\left(s_{i}\right)\right) \Delta s_{i-1}-\left(\kappa\left(s_{i}\right)-\kappa\left(s_{i-1}\right)\right) \Delta s_{i}}{\Delta s_{i-1, i} \Delta s_{i-1} \Delta s_{i}},
\end{gathered}
$$

where $\Delta s_{i, j}=\frac{\Delta s_{i}+\Delta s_{j}}{2}$. Since $\mathbf{D}_{2}$ is a matrix operator over a vector, we $\operatorname{stack}^{2}(7)$ from $i=1, \ldots, H$ where $H$ is the prediction horizon and obtain (1).

Similarly to $\mathbf{D}_{2}, \mathbf{D}_{1}$ is obtained by stacking (6),

$$
\mathbf{D}_{1}=\left[\begin{array}{ccccc}
\frac{1}{\Delta s_{0}} & -\frac{1}{\Delta s_{0}} & & & \\
& \frac{1}{\Delta s_{1}} & -\frac{1}{\Delta s_{1}} & & \\
& & \ddots & \ddots & \\
& & & \frac{1}{\Delta s_{H-1}} & -\frac{1}{\Delta s_{H-1}}
\end{array}\right]
$$

The problem formulation (5) is not in convex form due to the fact that the reconstructed path $(\mathbf{x}(\boldsymbol{\kappa}), \mathbf{y}(\boldsymbol{\kappa}))$ is computed using (4), which are clearly nonlinear equations. A linear approximation is done using a first-order Taylor approximation of both cosine and sine around the path orientation $\boldsymbol{\theta}^{\text {ref }}$. The linearization around $\boldsymbol{\theta}^{\text {ref }}$ takes the form

$$
\begin{aligned}
& \mathbf{x}(\boldsymbol{\kappa})=\mathbf{S}\left(\cos \left(\boldsymbol{\theta}^{\text {ref }}\right)-\sin \left(\boldsymbol{\theta}^{\text {ref }}\right) \odot\left(\mathbf{S} \boldsymbol{\kappa}-\boldsymbol{\theta}^{\text {ref }}\right)\right) \\
& \mathbf{y}(\boldsymbol{\kappa})=\mathbf{S}\left(\sin \left(\boldsymbol{\theta}^{\text {ref }}\right)+\cos \left(\boldsymbol{\theta}^{\text {ref }}\right) \odot\left(\mathbf{S} \boldsymbol{\kappa}-\boldsymbol{\theta}^{\text {ref }}\right)\right),
\end{aligned}
$$

where $\odot$ is the Hadamard product. Also, the problem may be infeasible when there is no solution that respects the constraints (5b) and (5c). Therefore, we add a slack variable $\boldsymbol{\Delta}=\left[\begin{array}{ll}\boldsymbol{\Delta}_{x} & \boldsymbol{\Delta}_{y}\end{array}\right]^{\top}$ and we minimize the cost function taking the slack variable into account as well. Also, with the inclusion of the slack variable $\Delta$, the deviations from the path are explicitly included in the cost function which enforces convergence to the reference path. In conclusion, the EMPC problem is formulated to yield the linear function with the smallest slope that produces a clothoid-like vehicle motion respecting the constraints. The final formulation is

$$
\begin{array}{ll}
\min _{\boldsymbol{\kappa}, \boldsymbol{\Delta}} & || \mathbf{D}_{2} \boldsymbol{\kappa}\left\|_{2}^{2}+\alpha\right\| \mathbf{D}_{1} \boldsymbol{\kappa} \|_{2}^{2}+\boldsymbol{\Delta}^{\top} \boldsymbol{\Lambda} \boldsymbol{\Delta} \\
\text { s. t. } & \left|\mathbf{x}(\boldsymbol{\kappa})-\mathbf{x}_{\text {ref }}\right| \leq \boldsymbol{\varepsilon}_{x}+\boldsymbol{\Delta}_{\boldsymbol{x}} \\
& \left|\mathbf{y}(\boldsymbol{\kappa})-\mathbf{y}_{\text {ref }}\right| \leq \boldsymbol{\varepsilon}_{y}+\boldsymbol{\Delta}_{\boldsymbol{y}} \\
& \left|\mathbf{D}_{1} \boldsymbol{\kappa}\right| \leq \mathbf{1} c_{\max } \\
& |\boldsymbol{\kappa}| \leq \mathbf{1} \kappa_{\max } \\
& \boldsymbol{\Delta} \geq 0 \\
& \kappa_{0}=\kappa_{\text {vehicle }},
\end{array}
$$

where $\boldsymbol{\Lambda} \in \mathbb{R}^{2 H \times 2 H}$ is a diagonal matrix of the form $\operatorname{diag}\left(\lambda_{1}, \ldots, \lambda_{H}, \lambda_{1}, \ldots, \lambda_{H}\right)$ that penalizes constraint violations.

The final formulation of the controller is trivially cast as a quadratic program (QP) by defining the optimization variable $\mathbf{u}=[\boldsymbol{\kappa}, \boldsymbol{\Delta}]^{\top}$.

\section{Constraint penalization}

We constrain the vehicle's predicted position to be inside of a box around the waypoint. By designing an off-road driving controller we can interpret the dimension of this constraint as the vehicle's desired maximum deviation from the path. In case of road driving, this is equivalent to the lane width minus half of the vehicle width. Hence, it is crucial to understand how the tuning parameters $\varepsilon$ and $\boldsymbol{\lambda}$ influence the driving experience. The first one determines the size of the box constraints and consequently how much freedom the controller has to choose a suitable curvature function such that the predicted path is within these constraints. The latter parameter sets how flat (or how sharp) the quadratic penalization $\|\boldsymbol{\Delta}\|_{2}^{2}$ is when the predicted path violates the box constraints. 


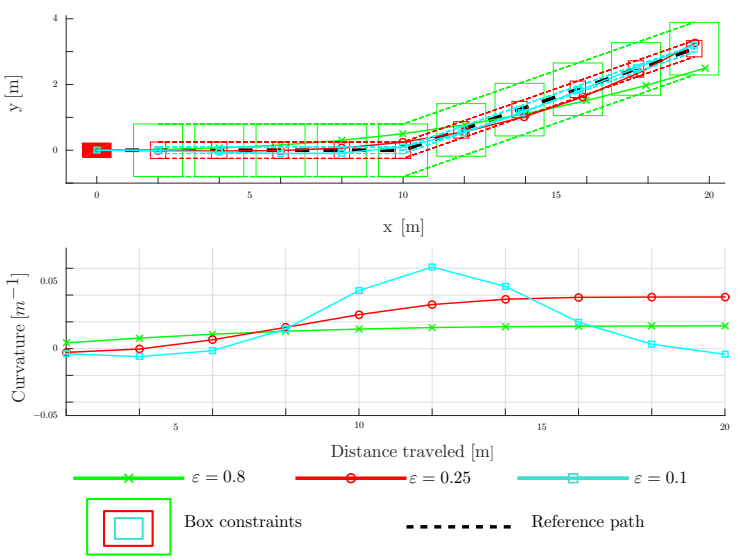

Fig. 2. Simulation of one run of the controller with $H=10$ where the size box constraint $\varepsilon$ is varied.

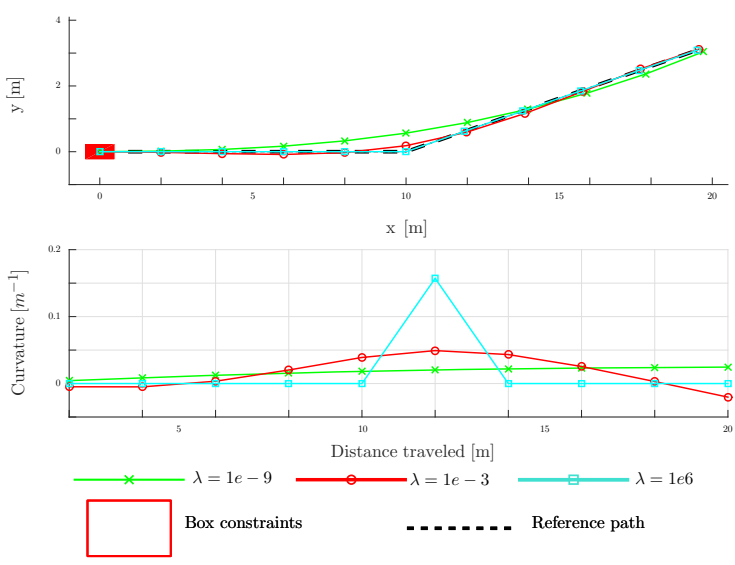

Fig. 3. Simulation of one run of the controller with $H=10$ where the penalization to violate the constraint $\boldsymbol{\lambda}$ is varied. In this case, $\boldsymbol{\lambda}$ is constant along the horizon.

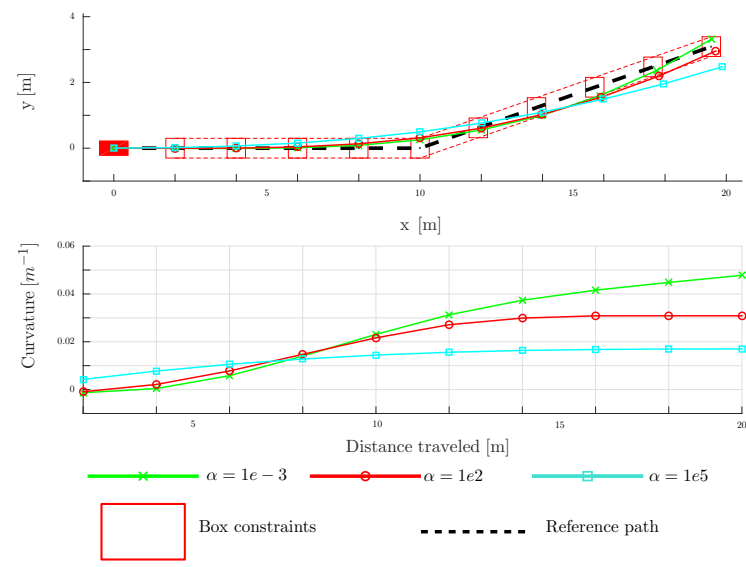

Fig. 4. Simulation of one run of the controller with $H=10$ where the importance given to the first derivative of curvature $\alpha$ is varied.

In Fig. 2 and 3, the influence of the parameters $\varepsilon$ and $\lambda$ are depicted, respectively. In Fig. 2, we see the effect of the size of the box constraints. The larger the box constraint, the closer to linear the curvature function is. In Fig. $3, \lambda$ is

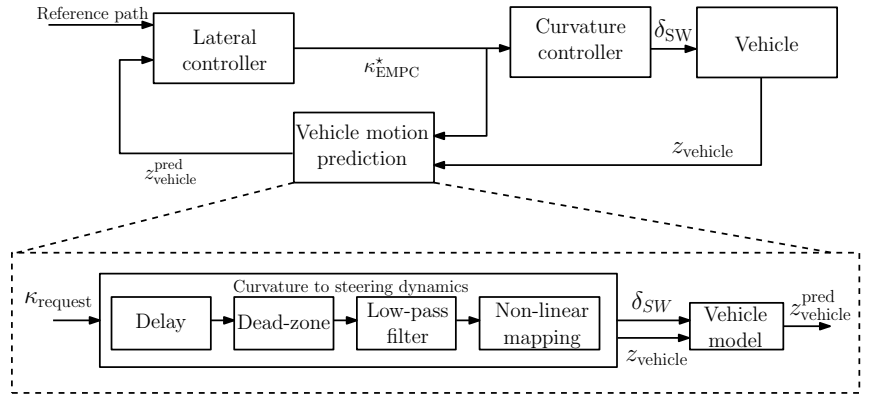

Fig. 5. Block diagram of the control algorithm implemented in the vehicle.

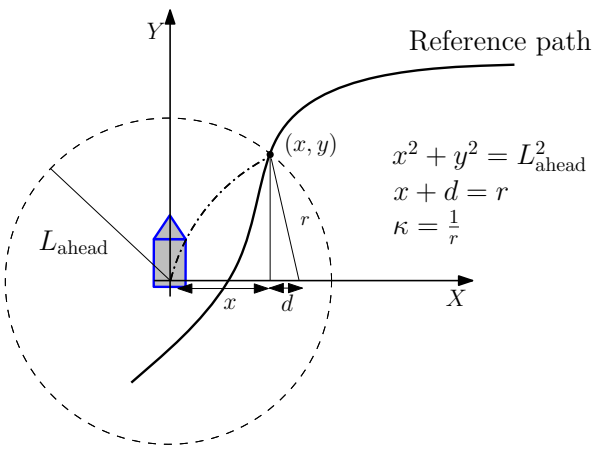

Fig. 6. The pure-pursuit controller illustration (inspired by [19])

constant over the horizon and we study how the curvature function changes for different values of $\boldsymbol{\lambda}$. The smaller $\boldsymbol{\lambda}$ is, the closer to linear the curvature function is. However, the predicted vehicle path can lay outside the constraint boxes with low penalization. In the extreme case $\boldsymbol{\lambda}=0$, the curvature function is the current vehicle curvature throughout the horizon.

\section{First derivative penalization influence}

Another parameter to consider in the controller parameter tuning is $\alpha$, which sets the importance of maintaining the current constant curvature throughout the horizon. Fig. 4 depicts the influence of $\alpha$ on the curvature function. The larger $\alpha$ is, the more constant the curvature function is. We can see that if we penalize $\left\|\mathbf{D}_{1} \boldsymbol{\kappa}\right\|_{2}^{2}$ more, the variation in the curvature decrease. This is an intended property of the controller, since the term was included to regularize the amount of steering that we need in order to follow the path This way it is possible to favour changing the curvature as little as possible.

\section{RESULTS}

In this section, we describe the experimental results obtained by deploying the EMPC in a Scania construction truck. Moreover, the EMPC is benchmarked against a purepursuit controller (PPC) that was previously deployed on the truck and is developed by Scania CV AB. We briefly describe the PPC and its idea. Afterwards, we detail the test scenario used in the experimental evaluation and the controller architecture.

\section{A. Pure-pursuit controller}

Let the desired curvature be the vehicle input. Fig. 6 depicts an illustration of the pure-pursuit controller algorithm [19]. In fact, this method is inspired by the way humans 
drive. Humans tend to look some distance in front of the car and head towards that spot. The algorithm has only one userdefined parameter (i.e., look-ahead distance $L_{\text {ahead }}>0$ ) with which the goal position is chosen. With the goal position defined, simple geometric relations are used to determine the curvature required, represented by $\kappa$ in Fig. 6, to move the vehicle from its current position to the goal position, denoted by $(0,0)$ and by $(x, y)$, respectively, in Fig. 6 .

The pure-pursuit controller is known due to its versatility and ease of implementation. However, a fine tuning is hard to accomplish and it is vehicle specific. Furthermore, the main parameter tuning, the look-ahead distance, has a large influence on in the path following accuracy, which can never obtain null deviation in curvy roads, resulting in a major drawback of the method.

\section{B. Scenario}

The reference path is recorded at Scania's test tracks facilities near Södertälje, Sweden. The reference path used is generated by recording a GPS trace using a real time kinematic GPS installed in the truck. The paths are then subsampled and consist of a waypoint sequence, in which the waypoints are two meters apart. We use a precision track, which is a challenging gravel road designed to perform autonomous mining truck tests, since it resembles a mining site. It is a narrow road with an approximate length of $1.5 \mathrm{~km}$. It has sharp turns where consequently the vehicle speed can not be high. Therefore, it is the perfect scenario to evaluate the performance of control algorithms designed to be extremely accurate at low speeds. Fig. 7 shows a video frame from the experiment performed at the precision track. The road is extremely narrow at some points, as at the position represented in Fig. 7, where the vehicle has just little more space than its own width. In mining sites, the roads are expected to be similar to this one, which motivates this experiment and the crucial need for high accuracy.

\section{Control architecture}

In Fig. 5, the block diagram of the control algorithm implemented in the vehicle is illustrated. As can be seen, the information fed to the controller is, besides the reference path, the predicted state of the vehicle. This strategy is necessary to handle the effect of the steering dynamics. These dynamics cause a non-neglectable delay between the curvature request and the actual steering actuation. The curvature of the predicted path provided by EMPC is fed to a curvature controller. There, a simple PI controller is used to compute the steering wheel angle request that tracks the computed curvature profile.

Both the PPC and EMPC were implemented in MATLAB/Simulink and the optimization problem is solved using cvxgen, which is a fast custom optimization solver [20].

\section{Experimental results using a Scania construction truck}

The experimental results are shown in Fig. 8 and 9, where the EMPC is deployed on a Scania construction truck, and we benchmark it against the PPC. The truck used in the experimental evaluation is a modified Scania G480 construction truck and it is shown in Fig. 1. The vehicle is equipped with a sensor platform and a servo motor for automated control of the steering column. An RTK-GPS is used for positioning during the experiments.

In Fig. 8, we compare the curvature requests from the PPC and the EMPC and their lateral deviation from the path. The results are summarized in the Table II. We also provide a

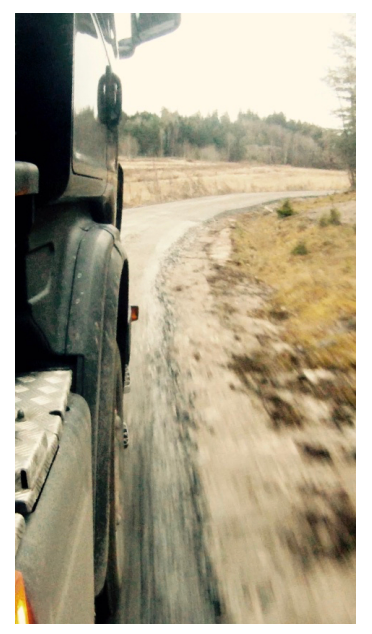

Fig. 7. Right front wheel of the truck, while performing the precision track The lack of space between the vehicle and the side of the road demonstrates how narrow the road is and how accurate the controller needs to be.

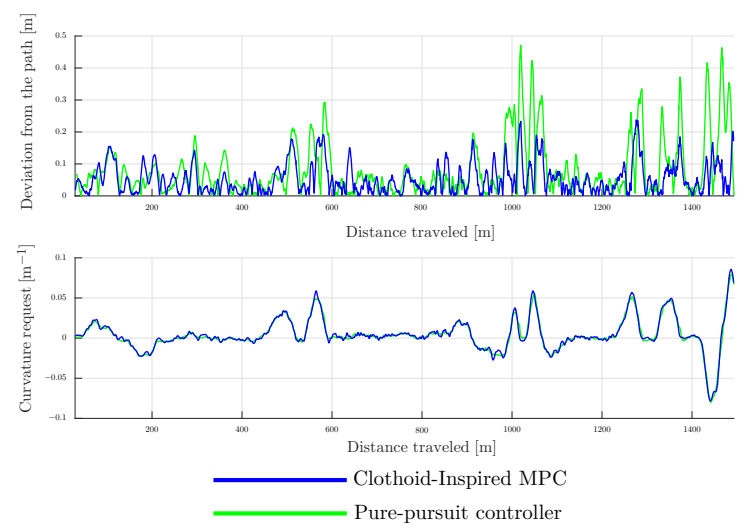

Fig. 8. Experimental run of the PPC and EMPC on the precision track. Above, it is depicted the controllers curvature requests. Below is shown the deviation from the path of both controllers.

statistical analysis about the distance to path in Fig. 9. In the boxes that are depicted in this figure, the central mark is the median, the edges of the box are the 25th and 75th percentiles and the whiskers extend to the most extreme data points not considered outliers. The meaning of the boxes is detailed in Fig. 10.

It is clear that the EMPC outperforms the PPC (see Fig. 8). The performance of both controllers is good with an average deviation from the path of less than $10 \mathrm{~cm}$ (see Table II). Moreover, the EMPC reduces by half the maximum and the average deviation, from the path when compared with the PPC. Therefore, the EMPC produces an extremely good path following accuracy.

\section{CONCLUSiOnS AND Future WORK}

In this paper, we present an economic model predictive controller (EMPC) for smooth lateral control of an autonomous truck. While in standard reference tracking MPC the cost function is zero at the reference path, in the EMPC the cost function is included an economic cost associated with the plant operation. In this case, the economic cost is driving comfort and smoothness. With this purpose, we minimize the second and first derivatives of the vehicle curvature. So, we encourage the vehicle to be guided using 

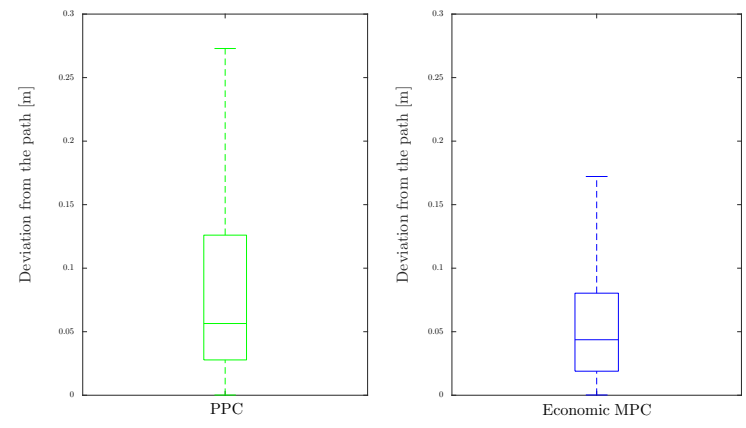

Fig. 9. Experimental run of the PPC and EMPC on the precision track. Statistical analysis of the deviation from the path for both controllers. The legend of the figure can be seen in Fig. 10.

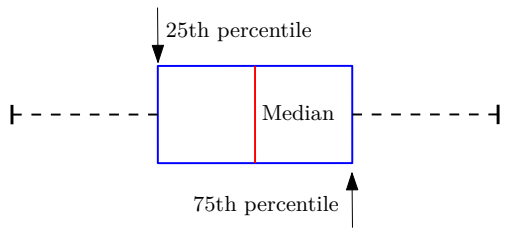

Fig. 10. Box plot legend. The central mark is the median, the edges of the box are the 25th and 75th percentiles and the whiskers extend to the most extreme data points not considered outliers.

linear-varying curvature in order to provide a smooth driving. Nevertheless, we added soft constraints to the formulation in order to always drive in the reference path vicinity. We concluded that smaller constraint slack penalization $\boldsymbol{\lambda}$ and bigger box constraints size $\epsilon$ contribute to a smoother driving and that the regularization parameter $\alpha$ smooths the curvature function depending on its magnitude. We experimentally benchmarked the EMPC against a PPC, both deployed in a Scania construction truck. The EMPC clearly outperformed the PPC and its performance is outstanding since the maximum deviation from the path never exceeds $30 \mathrm{~cm}$ and in average is $6 \mathrm{~cm}$. As future work, we will consider the development of a procedure to systematically tune the parameters of the controller, maybe even online, while driving. Furthermore, we should compare EMPC with other MPC-like controllers and also provide an analytical proof of the controller properties.

\section{REFERENCES}

[1] M. Harris, "Documents confirm Apple is building selfdriving car," The Guardian, 2015. [Online]. Available: http://www.theguardian.com/technology/2015/aug/14/apple-selfdriving-car-project-titan-sooner-than-expected

[2] J. Markoff, "Google cars drive themselves, in traffic," New York Times, 2010, October 9

[3] J. Funke, P. Theodosis, R. Hindiyeh, G. Stanek, K. Kritatakirana, C. Gerdes, D. Langer, M. Hernandez, B. Muller-Bessler, and B. Huhnke, "Up to the limits: Autonomous Audi TTS," in Proceedings of the IEEE Intelligent Vehicles Symposium, June 2012, pp. 541-547.

[4] J. Ziegler, P. Bender, M. Schreiber, H. Lategahn, T. Strauss, C. Stiller, T. Dang, U. Franke, N. Appenrodt, and C. Keller, "Making Bertha drive 2014 - An autonomous journey on a historic route," IEEE Intelligent Transportation Systems Magazine, vol. 6, no. 2, pp. 8-20, 2014.

[5] S. Thrun, M. Montemerlo, H. Dahlkamp, D. Stavens, A. Aron, J. Diebel, P. Fong, J. Gale, M. Halpenny, G. Hoffmann, K. Lau, C. Oakley, M. Palatucci, V. Pratt, P. Stang, S. Strohband, C. Dupont, C. Koelen, C. Markey, C. Rummel, J. van Niekerk, E. Jensen, P. Alessandrini, G. Bradski, B. Davies, S. Ettinger, A. Kaehler, A. Nefian, and P. Mahoney, "Stanley: The robot that won the DARPA grand challenge," Journal of Field Robotics, vol. 23, no. 9, pp. 661692, 2006.
TABLE I

CONTROLLERS PARAMETERS FOR EXPERIMENTAL EVALUATION.

\begin{tabular}{|c|c|c|}
\hline Parameters / Controllers & PPC & EMPC \\
\hline Look ahead time gap, $L_{\text {ahead }}$ & $1.2 \mathrm{~s}$ & \\
\hline Prediction horizon, $H$ & & 10 \\
\hline Path sampling time, $T_{s}$ & & $200 \mathrm{~ms}$ \\
\hline Controller frequency & $50 \mathrm{~Hz}$ & $50 \mathrm{~Hz}$ \\
\hline Slack penalization, $\lambda_{i}$ & & 20 \\
\hline First derivative penalization, $\alpha$ & & 9 \\
\hline Box constraints sizes, $\epsilon_{i}$ & & 0 \\
\hline
\end{tabular}

TABLE II

DEVIATION FROM THE PATH COMPARISON IN REAL EXPERIMENTS.

\begin{tabular}{|c|c|c|c|}
\hline & Max. [m] & Average [m] & Std. dev. [m] \\
\hline PPC & 0.48 & 0.10 & 0.10 \\
\hline EMPC & 0.24 & 0.06 & 0.05 \\
\hline
\end{tabular}

[6] A. Broggi, P. Cerri, S. Debattisti, M. Laghi, P. Medici, M. Panciroli, and A. Prioletti, "PROUD - public road urban driverless test: Architecture and results," in Proceedings of the IEEE Intelligent Vehicles Symposium, June 2014, pp. 648-654.

[7] T. Nothdurft, P. Hecker, S. Ohl, F. Saust, M. Maurer, A. Reschka, and J. Bohmer, "Stadtpilot: First fully autonomous test drives in urban traffic," in Proceedings of the IEEE/RSJ International Conference on Intelligent Robots and Systems, Oct. 2011, pp. 919-924.

[8] F. Borrelli, P. Falcone, and T. Keviczky, "MPC-based approach to active steering for autonomous vehicle systems," International Journal of Vehicle Autonomous Systems, vol. 3(2), pp. 265-291, 2005.

[9] P. Falcone, F. Borrelli, H. Tseng, J. Asgari, and D. Hrovat, "Linear time-varying model predictive control and its application to active steering systems: Stability analysis and experimental validation," International journal of robust and nonlinear control, vol. 18, no. 8, pp. $862-875,2008$

[10] J. Kong, M. Pfeiffer, G. Schildbach, and F. Borrelli, "Kinematic and dynamic vehicle models for autonomous driving control design," in Proceedings of the IEEE Intelligent Vehicles Symposium, June 2015, pp. 1094-1099.

[11] P. Falcone, F. Borrelli, H. Tseng, and D. Hrovat, "Experimental validation of nonlinear predictive algorithms for steering and braking coordination in limit handling maneuvers," in Proceedings of the International Symposium on Advanced Vehicle Control, August 2010.

[12] J. Rawlings, D. Angeli, and C. Bates, "Fundamentals of economic model predictive control," in Proceedings of the IEEE Annual Conference on Decision and Control, December 2012, pp. 3851-3861.

[13] J. Ma, J. Qin, T. Salsbury, and P. Xu, "Demand reduction in building energy systems based on economic model predictive control," Chemical Engineering Science, vol. 67, no. 1, pp. 92-100, 2012.

[14] T. Hovgaard, K. Edlund, and J. Jorgensen, "Economic MPC for power management in the smartgrid," in Proceedings of the European Symposium on Computer Aided Process Engineering, vol. 29, May 2011, pp. 1839-1843.

[15] P. Lima, M. Trincavelli, J. Mårtensson, and B. Wahlberg, "Clothoidbased model predictive control for autonomous driving," in Proceedings of the European Control Conference, July 2015, pp. 2983 - 2990.

[16] W. Kühn, Fundamentals of road design. WIT Press, 2013, vol. 20.

[17] H. Marzbani, R. Jazar, and M. Fard, "Better road design using clothoids," in Sustainable Automotive Technologies 2014. Springer International Publishing, 2015, pp. 25-40.

[18] P. Davis and P. Rabinowitz, Methods of numerical integration. Courier Corporation, 2007.

[19] R. Coulter, "Implementation of the pure-pursuit path tracking algorithm," Carnegie Mellon University, Tech. Rep., 1992.

[20] J. Mattingley and S. Boyd, "CVXGEN: A code generator for embedded convex optimization," Optimization and Engineering, vol. 13, pp. $1-27,2012$. 